\title{
Bandwidth Efficient Root Nyquist Pulses for Optical Intensity Channels
}

\author{
Sabeena FATIMA, S. Sheikh MUHAMMAD, A. D. RAZA
}

\author{
Dept. of Electrical Engineering, National University of Computer and Emerging Sciences, Lahore, Pakistan \\ sabeena.fatima@nu.edu.pk,sm.sajid@nu.edu.pk, ad.raza@nu.edu.pk
}

\begin{abstract}
Indoor diffuse optical intensity channels are bandwidth constrained due to the multiple reflected paths between the transmitter and the receiver which cause considerable inter-symbol interference (ISI). The transmitted signal amplitude is inherently non-negative, being a light intensity signal. All optical intensity root Nyquist pulses are timelimited to a single symbol interval which eliminates the possibility of finding bandlimited root Nyquist pulses. However, potential exists to design bandwidth efficient pulses. This paper investigates the modified hermite polynomial functions and prolate spheroidal wave functions as candidate waveforms for designing spectrally efficient optical pulses. These functions yield orthogonal pulses which have constant pulse duration irrespective of the order of the function, making them ideal for designing an ISI free pulse. Simulation results comparing the two pulses and challenges pertaining to their design and implementation are discussed.
\end{abstract}

\section{Keywords}

Nyquist Pulse, optical intensity signaling, PSWF (Prolate Spheroidal Wave Functions), MHPF (Modified Hermite Polynomial Function), ISI (Inter Symbol Interference)

\section{Introduction}

The capacity of Radio Frequency (RF) systems is limited due to scarcity and cost of licensing. Therefore, to meet the need of growing data rate requirements in wireless communications optical bands are being excessively exploited. Optical signals have numerous advantages over RF signals, the most attractive ones being that they are unlicensed and have many $\mathrm{THz}$ of bandwidth naturally associated to them.

Wireless optical intensity channel transmit information by modulating the instantaneous optical intensity. A Light Emitting Diode (LED) or a Laser Diode (LD) converts the input electrical signal to an optical signal. A photodiode detector at the receiver end converts the incident optical intensity signal back to an electrical signal. The optical intensity channel commonly employs Intensity Modulation and Direct Detection (IM/DD) [2]. Multipath distortion arising due to the multiple reflected paths between the transmitter and the receiver in indoor environment cause severe Inter Symbol Interference (ISI), necessitating the use of pulse shaping. Diffuse links [3] are attractive as they allow user mobility and eliminate the need of alignment but at the expense of bandwidth. The diffuse indoor optical links are severely bandwidth limited.

For indoor optical applications, signaling design varies significantly from the conventional electrical channels as optical channels impose the additional requirement of signal being non-negative. Secondly optical links require that for the case of indoor optical channels the transmitted signal be Class I eye safe under all conditions. As a result the peak transmitted power of the light signal remains constrained. Received signal is corrupted by free space losses, shot noise and ambient light noise. For the case of indoor optical channels distortion due to shot noise is most significant. Shot noise is modeled as uncorrelated additive white Gaussian noise [3].

There exists a wealth of literature on design of pulses that maximize in-band energy $[4,5,6]$ to mitigate the effect of ISI; however these pulses have been designed for the electrical channel. Halpern [7] proposed a design for finite duration Nyquist pulses for mean power constrained channels but not subject to amplitude constraints. Considerable information on optical intensity channels and their numerous advantages have been investigated [3, 8, 9]. However, the design of optical intensity pulse to minimize ISI still requires a much deeper study [9]. Steve Hranilovic [1] proved that all optical intensity root Nyquist pulses must be time limited to a single symbol interval. The time-limitedness of the root Nyquist pulse eliminates the possibility of finding a bandlimited pulse. Steve [1] proposed Prolate Spheroidal Wave pulse to be used since it is time limited and also has better spectral concentration among all time limited signals. For signals limited to a single symbol interval PSWF is the optimum signal choice $[7,11,12]$. From studies on pulse shaping techniques for time limited systems [13, 14, 15] Prolate Spheroidal Wave Functions (PSWF) and Modified Hermite Polynomial Function (MHPF) have emerged as potential pulse shaping functions as they provide the optimal spectral concentration. Individual discussion on these two contender pulses is widely available but no comparison between the two has been drawn. Design of a root Nyquist pulse 
which maximizes the in-band fractional energy has been proposed in [4, 7], in context of electrical domain. A linear combination of PSWF pulses proposed in [4] seeks to maximize the in-band fractional energy and to satisfy the Nyquist criteria. The problem was reduced to solving the linear objective function. This design can be extended to the optical domain by introducing an additional constraint of optical signal remaining non-negative.

In this work we propose root Nyquist pulse designs. The non-negativity constraint limits the root Nyquist pulses to be time-limited [1]. Although this eliminates the possibility of finding bandwidth limited root Nyquist pulses, potential still exists to find bandwidth efficient pulses for these channels. Pulses can be shaped such that most of the energy is concentrated in the band of interest [16]. Section 2 presents the discussion on root Nyquist pulse and shows that they are strictly time limited to a single symbol interval. Sections 3 and 4 focus on the discussion of PSWF and MHPF pulses. In Section 5 a comparison is drawn between the two pulses. Conclusions are presented in Section 6.

\section{Optical Intensity Root Nyquist Pulses}

For the case where receiver filter is matched to the transmitted pulse, to ensure zero ISI the pulse shape at the output must satisfy the Nyquist criteria [15] given by:

$$
\int x(\tau) x(\tau-k T) d \tau=\delta_{k 0}
$$

where $\delta_{k l}=\left\{\begin{array}{cc}1 & k=l \\ 0 & \text { otherwise. }\end{array}\right.$

An optical intensity root Nyquist pulse satisfies the non-negativity constraint in addition to the Nyquist criteria. All practical optical intensity root Nyquist pulses are timelimited to single symbol duration [1]. The root Nyquist pulse is required to be of small duration so that the number of interfering neighbors is small. This eliminates the possibility of obtaining band-limited root Nyquist pulses.

The search therefore reduces to bandwidth efficient pulses. Most of the energy needs to be concentrated in a finite band. The main objective is to maximize the in-band fractional energy of the time-limited root Nyquist pulse. Inband fractional energy is defined as the ratio of the energy of the pulse in the given bandwidth to the total energy of the time limited pulse. Its expression is given as [9]:

$$
\frac{\int_{-W}^{W} \mid X\left(\left.f\right|^{2} d f\right)}{\int_{-\infty}^{\infty} \mid X\left(\left.f\right|^{2} d f\right)}=K
$$

where $\mathrm{K} \in(0,1)$.

The $(1-\varepsilon)$ fractional energy bandwidth of a transmitted symbol $x(t)$ with Fourier Transform $X(f)$ is defined as [9]:
$W_{\varepsilon}(x)=W \varepsilon[0, \infty): \int_{-W}^{W}\left|X\left(\left.f\right|^{2} d f\right) \geq(1-\varepsilon) \int_{-\infty}^{\infty}\right| X\left(\left.f\right|^{2} d f\right)$

If $\varepsilon$ is chosen such that out of band energy is small, then $x(t)$ can be thought of as efficiently band-limited to $W \varepsilon(x)$.

The prolate spheroidal wave functions (PSWF) and modified hermite polynomial functions (MHPF) give the highest spectral concentration of all time limited signals $[14,15]$ and are discussed in detail in the next sections.

\section{Prolate Spheroidal Wave Functions}

Prolate spheroidal wave functions (PSWF) have the highest spectral concentration of all time-limited signals. They are strictly time-limited to $[0, T]$ and have maximum energy in the band of interest, i.e. $[-\Omega, \Omega]$ of all unit energy functions. These functions are solution to the integral equation [15]:

$$
\int_{-T / 2}^{T / 2} \frac{\sin \Omega(t-s)}{\pi(t-s)} \psi_{i}(s) d s=\lambda_{i} \psi_{i}(t)
$$

Here $\psi(\mathrm{t})$ represents the PSWF, $\lambda$ is the amount of energy contained in $[-T / 2, T / 2], \Omega$ is the bandwidth and $T$ is the pulse duration.

PSWF are an improvement on other pulses as they satisfy the double orthogonality property. This double orthogonality property guarantees unique demodulation at the receiver.

$$
\begin{gathered}
\int_{-\infty}^{\infty} \psi_{m}(t) \cdot \psi_{n}(t)=\delta_{m n}, \\
\int_{-T / 2}^{T / 2} \psi_{m}(t) \cdot \psi_{n}(t)=\lambda_{m} \delta_{m n}
\end{gathered}
$$

where $\psi_{n}(t)$ is the PSWF of order $n$.

$\psi_{n}(\mathrm{t})$ is written in terms of prolate angular function of first kind [15] as:

$$
\psi_{n}(t)=\psi_{n}(\Omega, T, t)=\frac{\left(2 \lambda_{n}(c) / T\right)^{1 / 2} S_{0 n}^{1}(c, 2 t / T)}{\left\{\int_{-1}^{1}\left[S_{0 n}^{1}(c, x)\right]^{2} d x\right\}^{1 / 2}}
$$

where $\left\{\int_{-1}^{1}\left[S_{0 n}^{1}(c, x)\right]^{2} d x\right\}^{1 / 2}=\frac{2}{2 n+1}$ where $S_{0 n}^{1}$ is the prolate angular function of first kind and $\lambda_{n}$ is the fraction of the energy of $\psi_{n}(\mathrm{t})$ that lies in the interval $[-1,1]$. by $[15]$

The prolate angular function of the first kind is given

$$
S_{0 n}^{1}(c, t)=\begin{array}{lll}
\sum_{k=0,2, \ldots}^{\infty} d_{k}(c) P_{k}(c, t) & n & \text { even } \\
\sum_{k=1,3, \ldots}^{\infty} d_{k}(c) P_{k}(c, t) & n & \text { odd }
\end{array}
$$

where $\mathrm{c}$ is the time bandwidth product given by $c=\Omega T / 2$, $P_{k}(c, t)$ is the Legendre polynomials [13][18] and $d_{k}(\mathrm{c})$ satisfy the recursion relation[14]:

$$
\alpha_{k} d_{k+2}^{n}(c)+\left(\beta_{k}-\chi_{n}(c)\right) d_{k}^{n}(c)+\gamma_{k} d_{k-2}^{n}(c)=0
$$

where the coefficients $\alpha, \beta, \gamma$ are given by: 


$$
\begin{gathered}
\alpha_{k}=\frac{(k+1)(k+2) c^{2}}{(2 k+3)(2 k+5)}, \\
\beta_{k}=\frac{\left(2 k^{2}+2 k-1\right) c^{2}}{(2 k-1)(2 k+3)}+k(k+1), \\
\gamma_{k}=\frac{(k)(k-1) c^{2}}{(2 k-1)(2 k-3)} .
\end{gathered}
$$

In order to compute $d_{k}^{n}(c)$ the following equation [15] is solved:

$$
O d^{n}=\chi_{n} d^{n}
$$

Here $d^{n}$ are the eigenvector and $\chi_{n}$ are the eigenvalues.

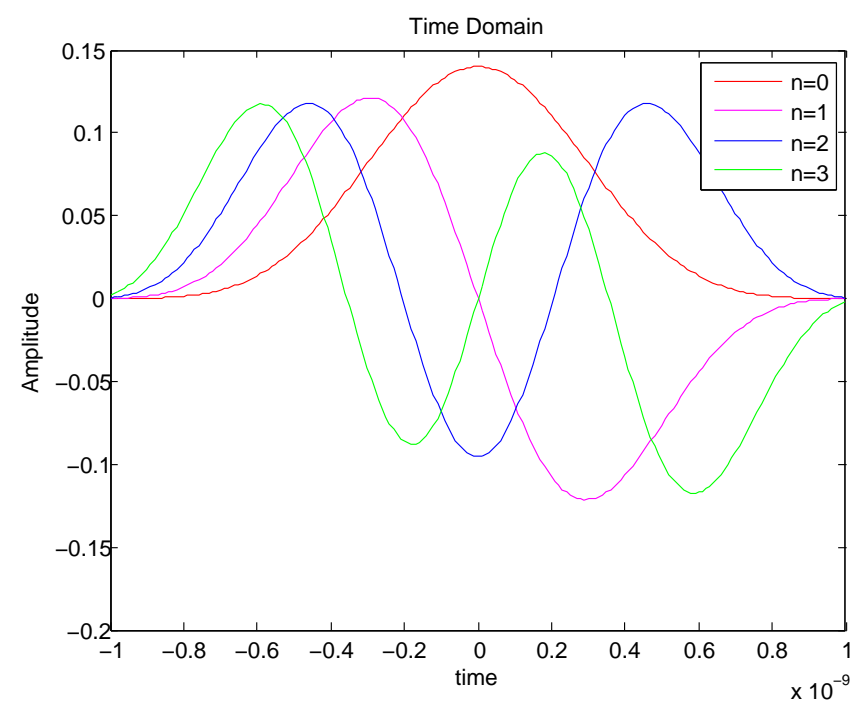

Fig. 1. Time Domain Representation of Prolate Spheroidal Wave Functions of order $0,1,2,3$. Note that the pulse duration is same for all orders and the number of zero crossings is equal to the pulse order.

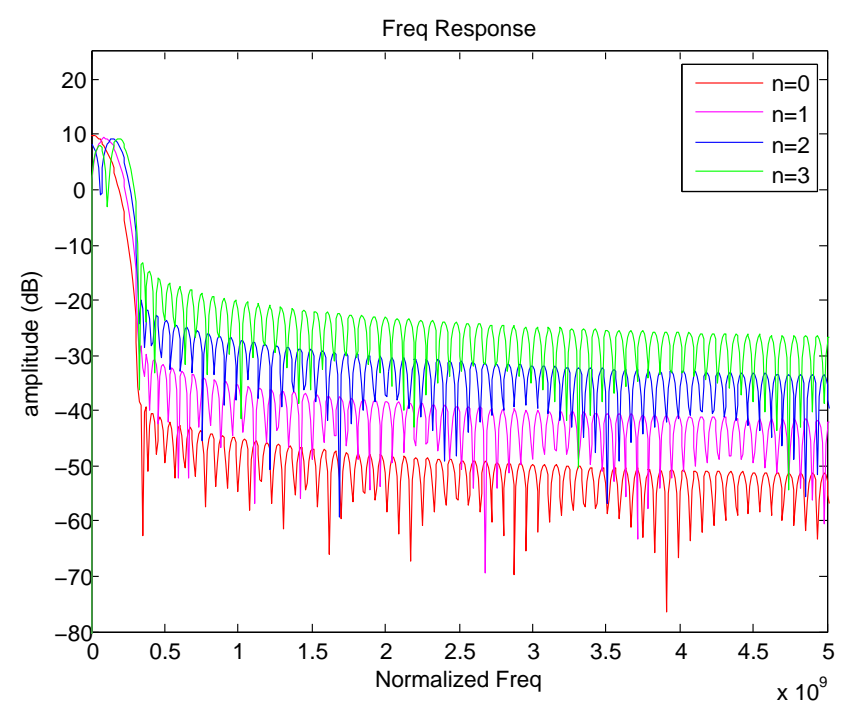

Fig. 2. Frequency Domain Representation of Prolate Spheroidal Wave Functions of order $0,1,2,3$.

Figures 1 and 2 represent the time and frequency characteristics for the PSWFs respectively. From the figures it can be deduced that the pulse duration and bandwidth is exactly the same for all orders $n$. Another interesting property being that number of zero crossings equals $n$. These pulses are double-orthogonal. Pulse duration and bandwidth can be bartered by utilizing the time-bandwidth product.

For wireless optical intensity channel only PSWF order 0 pulse can be utilized as it fulfills the amplitude nonnegativity constraint. However, making use of the interesting property of PSWF pulses that the number of zero crossings on time axis equals the pulse order $n$ and the pulse duration is same for all pulse orders, one approach could be to make a linear combination pulse of these orders and analyze its properties.

\subsection{Time Bandwidth Product}

To get a better understanding of time-bandwidth product $c$, PSWF order 0 pulse was plotted for different values of $c$. As time was kept confined to duration of 2 nanoseconds, the variation of $c$ had a direct impact on bandwidth. The $c$ value $\Omega T / 2$ effect the width of main lobe of PSWF pulse, as the $c$ value increases; the main lobe becomes compressed causing an expansion in frequency domain (as seen in the Figs. 3 and 4).

\subsection{Linear Combination Pulse}

Since we have limitations on channel bandwidth we need to concentrate most of the energy in our band of interest. We wish to maximize the in-band fractional energy which is given by:

$$
\frac{\int_{-W}^{W} \mid X\left(\left.f\right|^{2} d f\right)}{\int_{-\infty}^{\infty} \mid X\left(\left.f\right|^{2} d f\right)}=K
$$

Nigam [4] proposed a linear combination of PSWF pulses which maximize the in-band fractional energy. The linear combination pulse is given as:

$$
g(t)=\sum_{0}^{2 N+2} a_{j} \psi_{j}(t)
$$

Here $\psi_{j}(t)$ is the PSWF of $j$-th order; $N$ is the number of PSWFs used and $a_{j}$ are the coefficients which need to be determined.

Maximizing the in-band fractional energy reduces the problem [4] of determining the coefficients $a_{j}$ to

$$
\text { Maximize: } \sum_{0}^{2 N-2} a_{j} \psi_{j}(0) \lambda_{j}
$$

Subj to Nyquist Criteria:

$$
\sum_{0}^{2 N-2} a_{j} \psi_{j}(k T)=\delta_{k}
$$

This ensures that the overall pulse is zero at multiples of $T$ except $k=0$.

However, this pulse was designed for transmission in the electrical domain. Extending these results to the optical domain introduces an additional constraint that the transmitted pulse remains non-negative. Taking into account all 


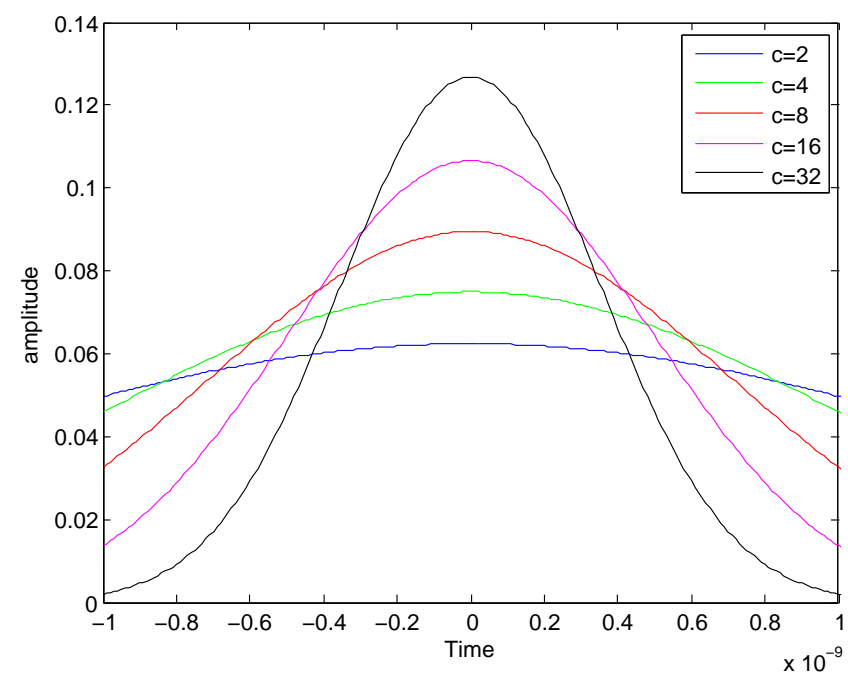

Fig. 3. Effect of varying time-bandwidth product $c$ (time representation).

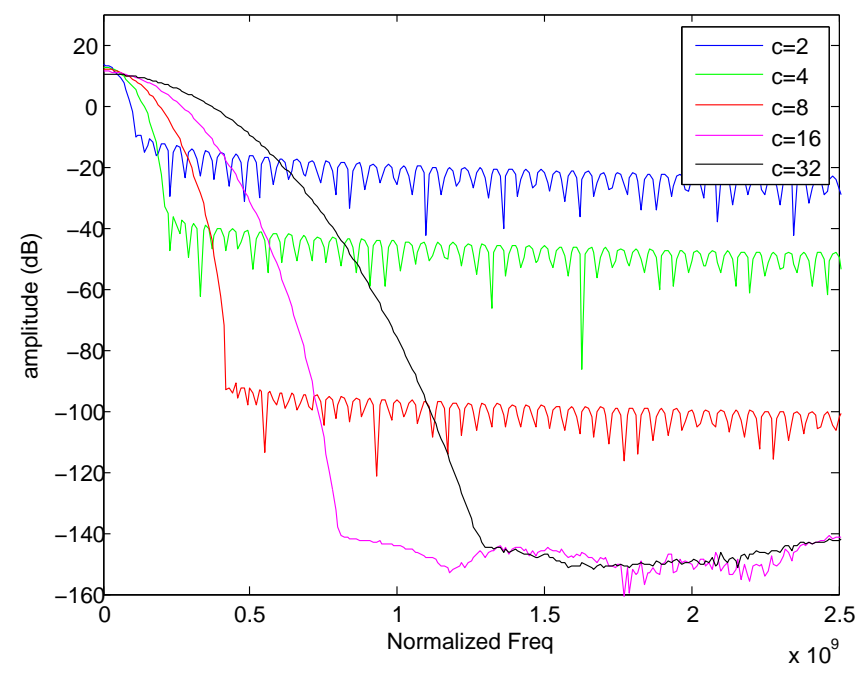

Fig. 4. Effect of varying time-bandwidth product (frequency representation). As the time-bandwidth product value increases the bandwidth occupied by the pulse increases.

these constraints the problem reduces to evaluating the linear objective function subject to linear constraints which can readily be solved by applying the simplex method. The results show that the coefficients for higher order PSWF pulse approach zero.

Firstly, we constrain the PSWF pulse to have unit value at time zero, then solving (13) and (14) for the coefficients $a_{j}$ we get the values shown in Tab. 1. As can be observed the PSWF order 0 pulse makes the major contribution to the linear combination pulse, in fact up to $c=8$ PSWF 0 pulse is the only contributor, beyond $c=8$ other even-ordered PSWF pulse shapes come into play. This proves that PSWF order 0 pulse is the optimum pulse which fulfills the non-negativity criteria.

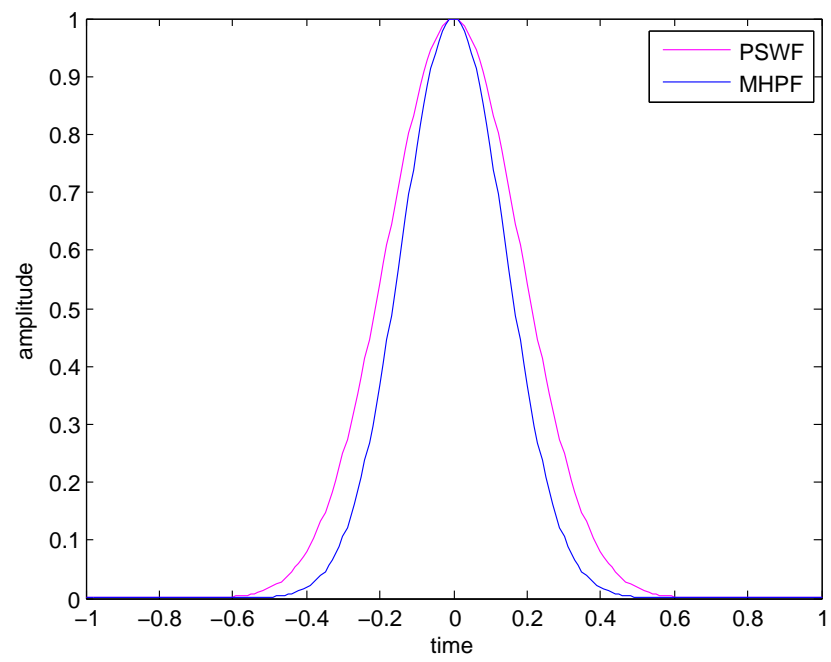

Fig. 5. Comparison between the optimal linear combination pulse and PSWF order 0 pulse proves that the optimal pulse follows the shape of the PSWF order 0 .

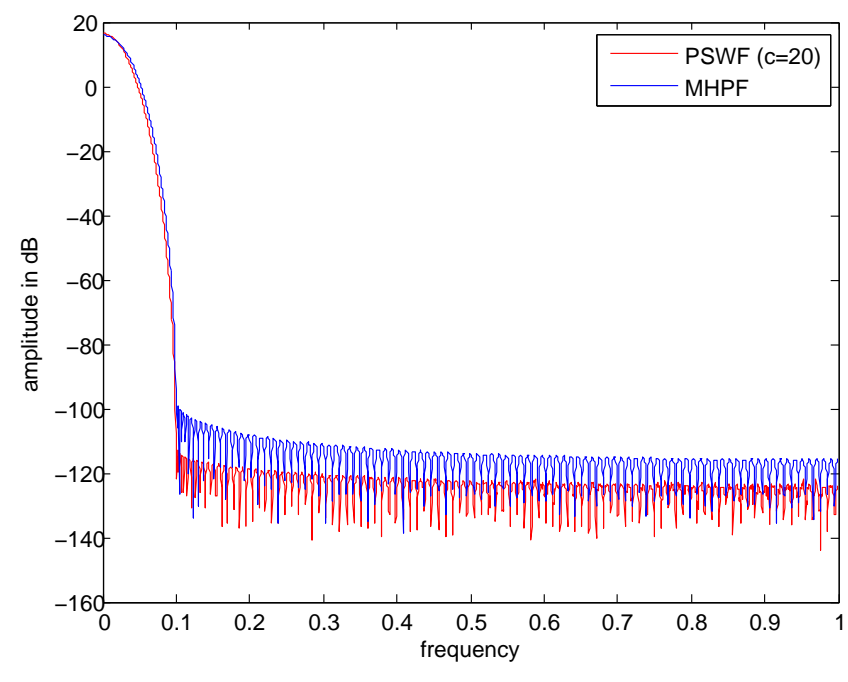

Fig. 6. No appreciable change in bandwidth between the two is observed proving that the major input is from the order 0 pulse in the optimal pulse design.

\begin{tabular}{ccccccc}
\hline \hline $\mathrm{c}$ & $a_{0}$ & $a_{1}$ & $a_{2}$ & $a_{3}$ & $a_{4}$ & $a_{5}$ \\
\hline 2 & 1 & 0 & 0 & 0 & 0 & 0 \\
4 & 1 & 0 & 0 & 0 & 0 & -0 \\
8 & 1 & 0 & 0 & 0 & 0 & -0 \\
10 & 0.9396 & 0 & 0.0860 & 0 & 0 & 0 \\
16 & 0.9442 & 0 & 0.0795 & 0 & 0 & 0 \\
20 & 0.9479 & 0 & 0.0668 & 0 & 0.0085 & -0 \\
\hline
\end{tabular}

Tab. 1. Coefficient values computed for the linear combination pulse for different time-bandwidth product using Simplex Methods. Note in order to ensure uniformity the PSWF pulses were scaled to have unit value at time 0 .

Figure 5 compares the optimal pulse with the PSWF order zero pulse, the linear combination pulse has a quicker decay rate and from the plot in Fig. 6 it can be observed that there is no significant change in band occupied by the two. 


\section{Modified Hermite Polynomial Function}

The Hermite polynomials are defined by [15][19]:

$$
h_{e n}(t)=(-1)^{n} e^{\frac{t^{2}}{2}} \frac{d^{n}}{d t^{n}}\left(e^{\frac{-t^{2}}{2}}\right)
$$

where $n=0,1,2, . .-\infty<t<\infty$. Hermite polynomials are not orthogonal [15]. However, they can be modified to become orthogonal as follows:

$$
h_{n}(t)=e^{\frac{-t^{2}}{4}} h_{e n}(t)=(-1)^{n} e^{\frac{-t^{2}}{4}} \frac{d^{n}}{d t^{n}}\left(e^{\frac{-t^{2}}{2}}\right) .
$$

The general formula for defining Modified Hermite Polynomial Functions (MHPF) is given by [15]:

$$
h_{n}(t)=k_{n} e^{\frac{-t^{2}}{4}} n ! \sum_{i=0}^{n / 2}\left(-\frac{1}{2}\right)^{i} \frac{t^{n-2 i}}{(n-2 i) ! i !} .
$$

The constant $k_{n}$ where $n=0,1,2, \ldots$ is indicative of the energy of the pulse given by [15]:

$$
k_{n}=\sqrt{\frac{E_{n}}{n ! \sqrt{2 \pi}}} .
$$

Figure 7 and Figure 8 represent the time and frequency response for the MHPFs. It can be observed that the pulse duration and bandwidth is exactly the same for different orders $n$ with the number of zero crossings in time domain being equal to $n$. These pulses are orthogonal to one another.

\section{Comparison}

The MHPF and PSWF are very different functions so drawing a comparison between the two is fairly complex. We confined the PSWF and MHPF pulse to similar time durations and observed the pulse shapes obtained.

PSWF pulse's duration and bandwidth can be bartered using a time-bandwidth product $c$ where $c=\Omega T / 2$. The MHPF pulses have no such constant $c$ so only one pulse shape is obtained for a given pulse duration. However, the MHPF pulse time duration and bandwidth are inversely related as $\Omega T / \pi$ [20].

The curves obtained when the MHPF and PSWF generated pulse shapes have identical time domain characteristics were plotted side by side to draw a better comparison. For instance, in the current scenario when the time bandwidth product $c=16$ with pulse duration $T=[-1,1]$, the MHPF and PSWF pulse have identical time domain characteristics (also shown in Fig. 9). While comparing the frequency domain plots obtained it was observed that the band occupied by the MHPF pulse is 1.3 times the band occupied by PSWF pulse which is roughly equal to $\pi / 2$ (Fig. 10).

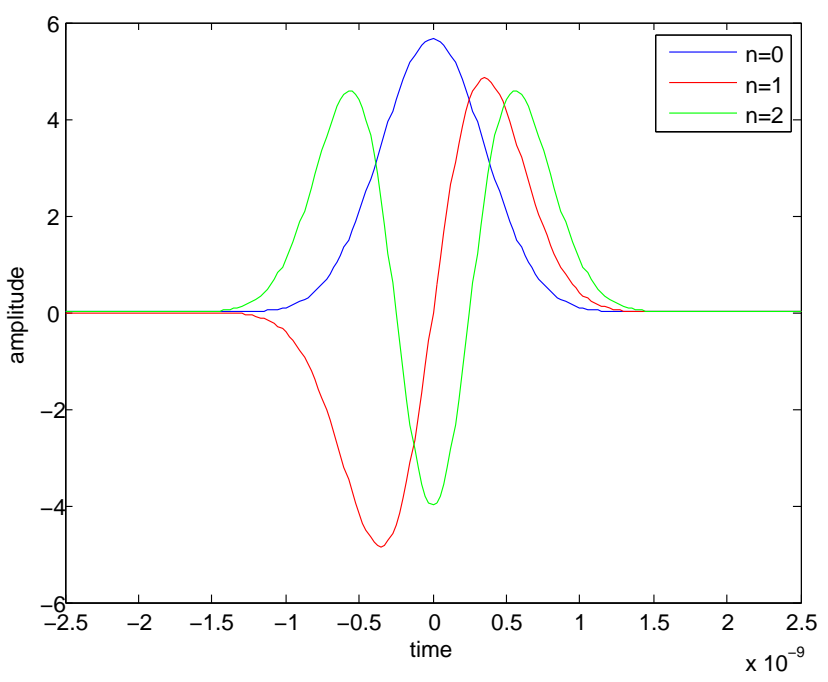

Fig. 7. Time domain representation of MHPF pulse for order 0 , 1,2. Different order pulses have identical pulse duration.

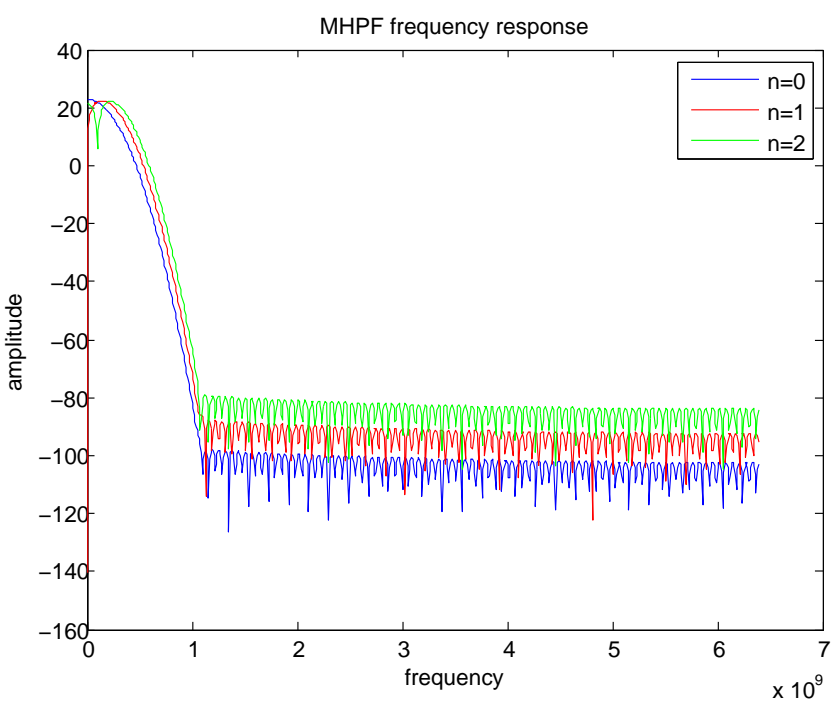

Fig. 8. Frequency representation for MHPF order 0, 1, 2 pulse. Note the constant identical bandwidth for different orders of the pulse.

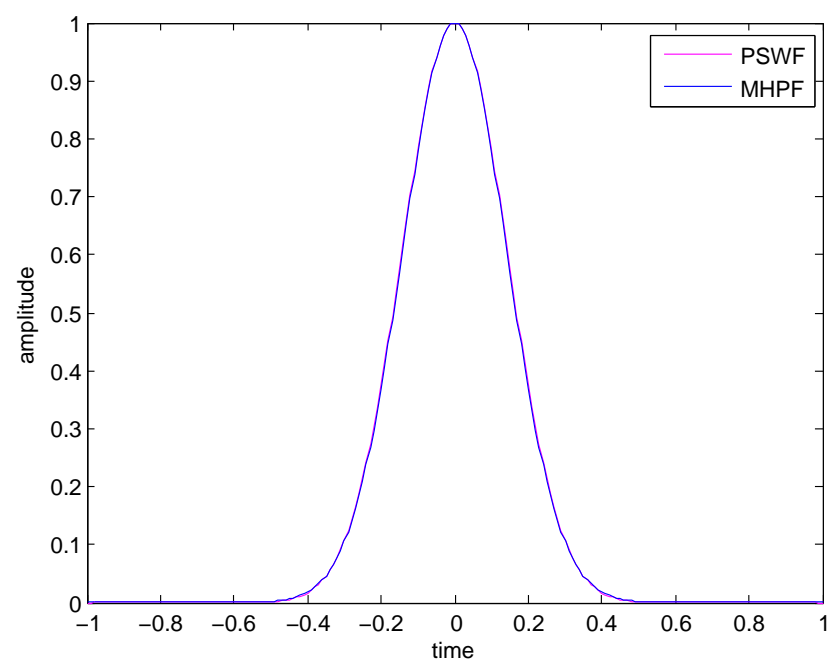

Fig. 9. PSWF $(c=16)$ and MHPF pulse. The two pulse shapes appear to be exactly similar. 


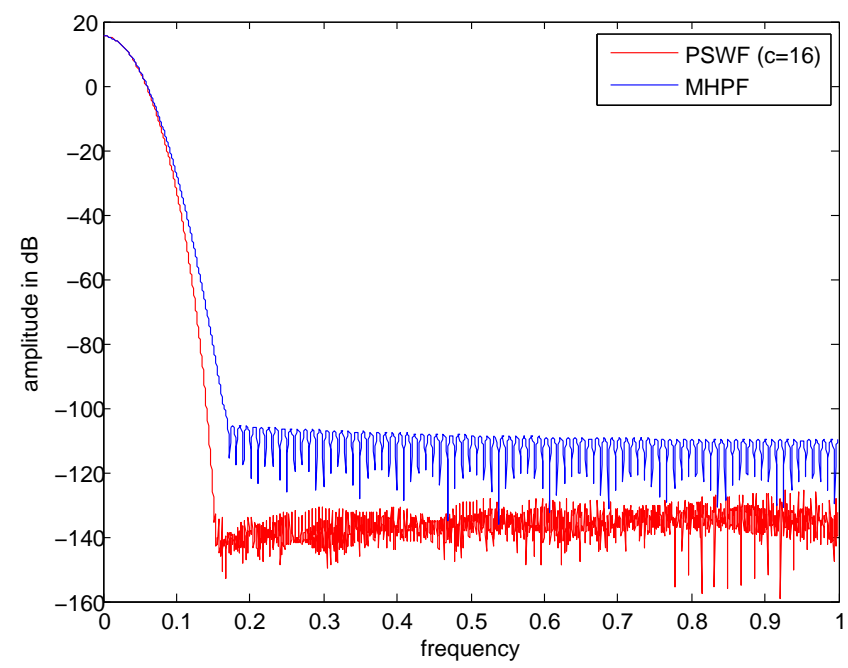

Fig. 10. Comparison between MHPF pulse and PSWF pulse $(c=16)$. Both occupy similar bandwidth as is expected from the similar time response.

Table 2 compares the band occupied by the PSWF and MHPF generated pulse while having similar parameters. cPSWF denotes the value of time-bandwidth product to obtain a PSWF pulse shape similar to MHPF for the same time-duration. It can be observed that the band occupied by MHPF pulse is $1.2-1.3$ times the one of PSWF pulse, therefore it can be safely said that the PSWF is most efficient in terms of bandwidth occupancy.

\begin{tabular}{ccccc}
\hline \hline Time & c-PSWF & BW-PSWF & BW-MHPF & Factor diff. \\
\hline$[-1,1]$ & 16 & 0.154 & 0.196 & $\simeq 1.3$ \\
{$\left[-\frac{3}{4}, \frac{3}{4}\right]$} & 10 & 0.1295 & 0.138 & $\simeq 1.1$ \\
{$\left[-\frac{1}{2}, \frac{1}{2}\right]$} & 4 & 0.081 & 0.104 & $\simeq 1.3$ \\
\hline
\end{tabular}

Tab. 2. Comparison of band occupancy of PSWF and MHPF generated pulse shapes under similar time duraction.

To validate the benefits of the pulse design technique utilized, optical data transmission in indoor environments has been simulated. The unequalized OOK system has been adapted for validation. The input bits, assumed to be independent identically distributed (i.i.d) and uniform on [0,1] are passed through a transmitter filter whose impulse response allows pulse shaping. The block diagram of the simulative setup is shown in Fig. 11 which uses the ceiling bounce model to cater for indoor light propagation [21]. The input signal $x(t)$ is passed through a multipath channel impulse response $h(t)$ and noise $n(t)$ is added. On the receiver end matched filter detection is employed as depicted in Fig. 11. To characterize the system performance, the eye diagram analysis (Fig. 12 and 13) has been utilized. The effect of multipath propagation and intersymbol interference is catered for better with our self designed pulse as the opening of the eye (Fig. 13) on an arbitrary amplitude scale is wider compared to the scenario where a rectangular pulse is used (Fig. 12).

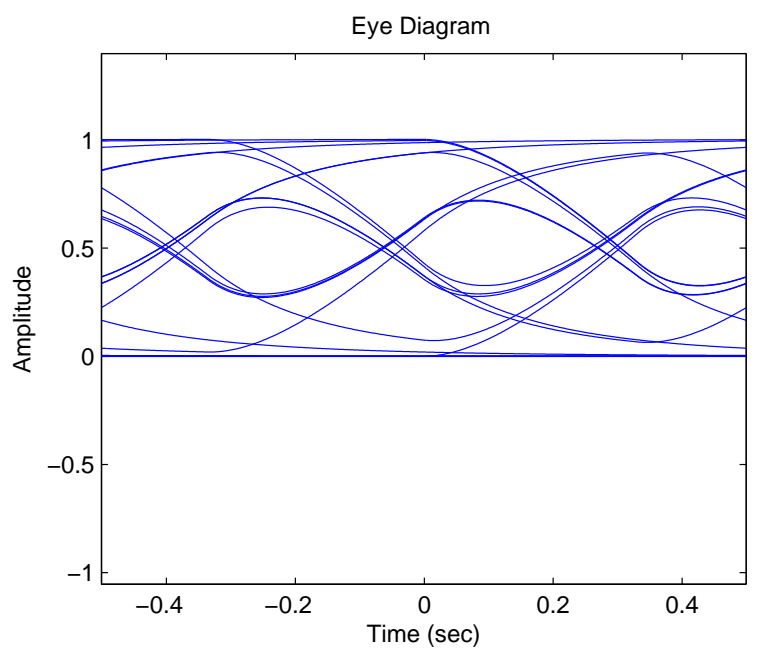

Fig. 12. Eye diagram using rectangular pulse for the Ceiling Bounce model.

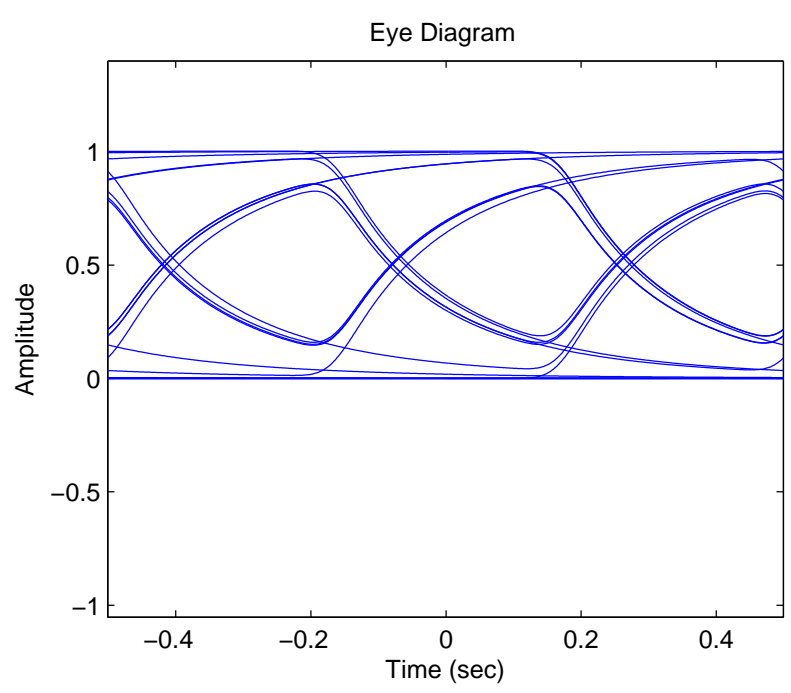

Fig. 13. Eye diagram using self designed pulse for the Ceiling Bounce model.

\section{Conclusion}

This paper investigates ISI free pulses satisfying the non-negative amplitude constraint of optical intensity channels for high speed optical wireless communications [22]. The optical intensity channels constrain the pulse to be nonnegative; also there is a limitation on channel bandwidth. This non-negativity constraint limits the root Nyquist pulse to be time limited [1]. Modified Hermite polynomial and Prolate Spheroidal wave functions have been proposed as potential pulse shaping functions. Both are time-limited and have most of the energy concentrated in a finite band. PSWF pulses' time and bandwidth can be bartered using a constant $c$ (the time-bandwidth product i.e. $\Omega T / 2$ ). Varying values for $c$ it is possible to arrive at a point where the MHPF and PSWF pulse have identical curves for any given time-duration. Comparing the bandwidth occupancy 


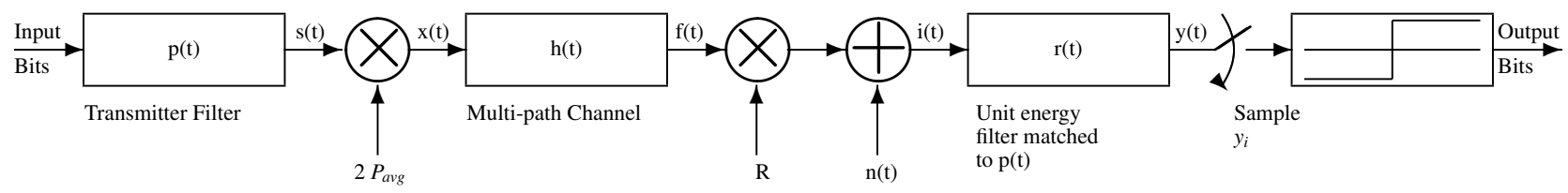

Fig. 11. Block diagram for the simulation setup.

of MHPF and PSWF under this scenario it was observed that the MHPF pulse occupies a band roughly 1.2 times the band occupied by the PSWF pulse. For a major segment (range of $c$ values), the MHPF pulse occupies more than twice the band when compared to the PSWF pulse. Also from optimizing the linear objective function PSWF order 0 pulse emerges as most efficient in terms of bandwidth concentration. MHPF pulses hold the advantage of being easier to generate as they have a closed form expression, contrary to the PSWF pulse which lack a closed form solution. Since both PSWF and MHPF pulses satisfy the optical channel constraints, they are well suited for ease of implementation and fulfill system bandwidth requirements.

\section{References}

[1] HRANILOVIC, S. Minimum bandwidth optical intensity nyquist pulses. IEEE Transactions on Communications, 2007, vol. 55, no. 3, p. 574-583. DOI: 10.1109/TCOMM.2006.888878

[2] HRANILOVIC, S., KSCHISCHANG, F. R. Optical intensitymodulated direct detection channels: signal space and lattice codes. IEEE Transactions on Information Theory, 2003, vol. 49, no. 6, p. 1385-1399. DOI: 10.1109/TIT.2003.811928

[3] KAHN, J. M., BARRY, J. R. Wireless infrared communications. Proceedings of the IEEE, 1997, vol. 85, no. 2, p. 263-298. DOI: $10.1109 / 5.554222$

[4] NIGAM, G., SINGH, R., CHATURVEDI, A. K. Finite duration root nyquist pulses with maximum in-band fractional energy. IEEE Communications Letters, 2010, vol. 14, no. 9, p. 797-799. DOI: 10.1109/LCOMM.2010.09.100314

[5] BEAULIEU, N.C., TAN, C.C., DAMEN, M.O. A better than Nyquist pulse. IEEE Communications Letters, 2001, vol. 5, no. 9, p. 367-368. DOI: $10.1109 / 4234.951379$

[6] SOOD, R., XIAO, H. Root Nyquist pulses with an energy criterion. In IEEE International Conference on Communications. Glasgow (UK), 2007, p. 2711-2716. DOI: 10.1109/ICC.2007.450

[7] HALPERN, P. Optimum finitie duration nyquist signals. IEEE Transactions on Communications, 1979, vol. 27, no. 6, p. 884-888. DOI: 10.1109/TCOM.1979.1094486

[8] RAMIREZ-INIGUIEZ, R., GREEN, R. J. Indoor optical wireless communications. In IEEE Colloquium on Optical Wireless Communications. London (UK), 1999, p. 14/1-14/7. DOI: 10.1049/ic:19990705

[9] TAVAN, M., AGRELL, E., KAROUT, J. Bandlimited intensity modulation. IEEE Transactions on Communications, 2012, vol. 60 , no. 11, p. 3429-3439. DOI: 10.1109/TCOMM.2012.091712.110496
[10] HRANILOVIC, S. Wireless Optical Communication Systems. Boston (USA): Springer Science + Business Media, 2005. DOI: $10.1007 / \mathrm{b} 99592$

[11] SELPIAN, D., POLLAK, H. O. Prolate spheroidal wave functions, fourier analysis and uncertainty - I. Bell Systems Technical Journal, 1961, p. 43-63.

[12] SELPIAN, D., POLLAK, H. O. Prolate spheroidal wave functions, fourier analysis and uncertainty - II. Bell Systems Technical Journal, 1962, p. 65-84.

[13] ALLEN, B., GHORASHI, S. A., GHAVAMI, M. A review of pulse design for impulse radio. In Proceedings of IEEE Ultra Wideband Communications Technologies and System Design. 2004, p. 93-97.

[14] GHAVAMI, M., MICHAEL, L., KOHNO, R. Hermite function based orthogonal pulses for ultra wideband communication. Wireless Personal Multimedia Communications Symposium, 2001.

[15] DILMAGHANI, R. S., GHAVAMI, M., ALLEN, B., AGHVAMI, H. Novel UWB pulse shaping using prolate spheroidal wave functions. In Proceedings of IEEE Personal, Indoor and Mobile Radio Communications. Beijing (China), 2003, vol. 1, p. 602-606. DOI: 10.1109/PIMRC.2003.1264343

[16] TAVAN, M., AGRELL, E. , KAROUT, J. Strictly bandlimited ISI-free transmission over intensity-modulated channels. In IEEE Globecom. Houston (TX, USA), 2011, p. 1-6. DOI: 10.1109/GLOCOM.2011.6133734

[17] PROAKIS, J. G. Digital Communications. $4^{\text {th }}$ ed. New York: McGraw-Hill, 2001.

[18] TSAI, C. Y., JENG, S. K. Design of Legendre polynomial based orthogonal pulse generator for ultra wideband communications. In IEEE Antennas and Propagation Society International Symposium. 2005, vol. 2B, p. 680-683. DOI: 10.1109/APS.2005.1552105

[19] GHAVAMI, M., MICHAEL, L.B., KOHNO, R. Ultra Wideband Signals and Systems in Communication Engineering. $2^{\text {nd }}$ ed. Wiley, 2007.

[20] OUERTANI, M., BESBES, H., BOUALLEGUE, A. Modified Hermite functions for designing new optimal UWB pulse-shapers. In European Signal Processing Conference (EUSIPCO). Antalya (Turkey), 2005.

[21] GHASEEMLOOY, Z., POPOOLA, W., RAJBHANDARI, S. Optical Wireless Communications: System and Channel Modelling with Matlab. London: CRC Press, 2013.

[22] TAVAN, M., AGRELL, E., KAROUT, J. Indoor high-speed optical wireless communications: Recent developments. In Proceedings of IEEE International Conference on Transparent Optical Networks (ICTON). Graz (Austria), 2014. 


\begin{abstract}
About the Authors...
Sabeena FATIMA received her BS in Telecommunications Engineering and MS in Electrical Engineering from the National University of Computer and Emerging Science, Lahore, Pakistan, in 2009 and 2013 respectively. She joined National University of Computer and Emerging Science in the year 2009 where she currently works as lecturer in Dept of Electrical Engineering. Her research interests include optical communications, signal estimation and detection techniques, MIMO systems and adaptive signal processing.
\end{abstract}

S. Sheikh MUHAMMAD completed his Bachelors in Electrical Engineering with Honors in 2001 and received Masters in Electrical Engineering in 2003 the from University of Engineering and Technology, Lahore, Pakistan whereby he also remained in the faculty for around 3 years. He completed his $\mathrm{PhD}$ in Electrical Engineering in 2007 with Excellence at the Graz University of Technology conducting research on coded modulation techniques for free space optical systems. He has Guest Edited 2 special issues in Optical Wireless Communications in 2009 and 2012 and has organized regular IEEE Colloquiums on Optical Wireless in 2008 (Austria), 2010 (UK) and 2012 (Poland). He has published more than 50 peer reviewed papers in journals and conferences of repute and has chaired number of international conference sessions. He is currently working as an Associate Professor of Electrical Engineering at the National University of Computer and Emerging Sciences (FAST-NU) in Lahore and his current research revolves around application of network information theory to optical wireless and optical wave propagation through random media.

A. D. RAZA received his Bachelors in Electrical Engineering with Honors in 1973 and Masters in Electrical Engineering in 1984 from the University of Engineering and Technology, Lahore, Pakistan. He remained with Pakistan's state owned Telecom operator from 1975 to 2008 and rose to the level of Executive Vice President. He joined the National University of Computer and Emerging Sciences in 2008 and is pursuing his doctoral studies besides his teaching responsibilities as an Assistant Professor in the Department of Electrical Engineering. His current research interest revolves around application of network information theory to optical wireless networks. 